\title{
Satisfaction assessment with Abbreviated Profile of Hearing Aid Benefit (APHAB) questionnaire on people using hearing aid having Real Ear Measurement (REM) eligibility
}

Selma Turan ${ }^{1}$ Selim Unsal ${ }^{2 *}$ Hanifi Kurtaran ${ }^{3}$

\begin{abstract}
Aim: Benefit and satisfaction from hearing aids can be measured in different ways. The aim of this study is to evaluate the benefits and satisfaction of the users from the hearing aids whose fitting are done suitably for hearing loss including ear mould.

Material and method: In this study Abbreviated Profile of Hearing Aid Benefit (APHAB) questionnaire which consists of 24 questions was used for hearing aid satisfaction assessment. Total 301 people having different type and degree hearing loss participated in the study. The ages of 141 men and 160 women participants were between 18-65 and the average age was $49.11 \pm 13.89$ year. 187 of the participants had hearing aid in only one ear and the 114 had in both ears. Degree of unilateral hearing loss was 31 slight, 64 moderate, 49 moderately severe, 35 severe and 8 profound. In the ear in which hearing aid was used, there was 134 sensorineural hearingloss, 53 mixture hearing loss. Degree of hearing loss of the people using hearing aid bilaterally was 24 slight, 79 moderate, 54 moderately severe, 48 severe and 23 profound. In 162 ears of people using hearing aid bilaterally there was sensorineural hearing loss and in 53 ears there was mixture hearing loss.
\end{abstract}

Results: In right ear average score of the maximum satisfaction was between 4.23-5.75 and in left ear it was between 4.20-5.72. While the degree of hearing loss was increasing, the average of satisfaction score was decreasing. In terms of using unilateral and bilateral hearing aid, statistically considerable difference was found. In terms of hearing loss statistically considerable difference couldn't be found.

Conclusion: Satisfaction of hearing aid is decreasing inversely proportional with increasing of hearing loss (slight- profound degree) While the furthest satisfaction for right and left ear was slight degree, the least satisfaction was observed for profound hearing loss. Aid using satisfaction changes depend on using the aid in the right ear or in the left ear. Using bilateral hearing aid has increased patient satisfaction much more.

Keywords: Hearing aid; hearing loss; satisfaction; left ear. 


\section{INTRODUCTION}

Hearing aids provide people with hearing loss to hear the noise and more importantly use the audio signals in the most effective way. They are the best implementations for the hearing losses which cannot be cured with medical or surgical operations ${ }^{1,2}$. Considering type, degree and configuration of hearing loss for choosing hearing aid increases the success of suitable and useful implementation. Besides, factors such as identification scores of speech in silent and noisy places, previous hearing aid experience, age, socio-cultural level, occupation, cosmetic concerns and expectations affect the benefit obtained from hearing aid ${ }^{2}$. Benefit obtained from hearing aid is measured in different ways. Among these there are free sound field measurements in silent and noisy places, speech recognition tests and questionnaries. It is necessary to evaluate the hearing aids objectively for these methods to achieve the goal. Evaluating the hearing aids objectively can be reached with Real Ear Measurement test (REM). Acoustic characteristics of the real-ear hearing aids are put forward by $\mathrm{REM}^{3}$. In terms of benefits and usefulness, questionnaire profiles used in the evaluation of hearing aids can be grouped in various ways. One of the most important of them is "Abbreviated Profile of Hearing Aid Benefit" (APHAB) ${ }^{4}$. APHAB is a hearing aid assessment questionnaire which is answered from the perspective of people who use hearing aids. It is questioning the individual's experience when using a hearing $a^{2}{ }^{5}$. The survey was developed considering that determining the difference between the responses given "with my hearing aid" and "without my hearing aid" and considering that determining advantages and disadvantages brought by using hearing aids would be possible. APHAB method is divided into four subgroups. It is assessing the different areas both the cases with hearing aids and without hearing aid. It consists of four subgroups and 24 questions. There are 6 questions in each subgroup. For each substance, the individual's own performance, the benefits of amplification, both with hearing aid and without hearing aid is available in the rating scale ${ }^{6-8}$. Compliance with various clinical studies of APHAP survey was conducted and formed the Chinese version of the APHAB survey and showed a high rate of satisfaction and evaluation could be reached ${ }^{9}$. The aim of this study is to evaluate the benefits and satisfaction of the patients whose earmould fitness and hearing aid benefits are confirmed by using Abbreviated Profile of Hearing Aid Benefit-Turkish (APHAB-TR) questionnaire.

\section{MATERIALS AND METHODS}

In this study Abbreviated Profile of Hearing Aid Benefit (APHAB) questionnaire which consists of 24 questions was used for hearing aid satisfaction assessment. Total 301 people having different type and rate hearing loss participated in the study. The ages of 141 men and 160 women participants were between 18-65 and the average age was $49.11 \pm 13.89$ year. 187 of the participants had hearing aid in only one ear and the 114 had in both ears. In this study, individuals have been selected from among the individuals whose hearing loss were determined by audio logical examination at any university, research hospital or state hospital and individuals used hearing aid at least 6 weeks. In addition, real-ear measurement tests of the individuals with hearing aids were made before the application of the questionnaire. People who are in the normal benefit curves were included in the study. Individuals who cannot reliably answer the questionnaire, whose real ear measurement is not within the normal benefit curve, having literacy problems, those with mental health problems, those are under 18 years of age and people over 65 were excluded from the study.

\section{Questionnaire application}

The patients were asked to fill in the questionnaire themselves. During this process, the researchers gave detailed information to those who participated in the questionnaire and all questions were answered from them in a descriptive manner. APHAB questionnaire consists of four subscales and measures of user satisfaction with the situation faced by patients in different situations reliably. Subscales of the questionnaire are Ease of Communication (EC), Reverberation (RV), Backround Noise (BN) and Adoption of Voice (AV).

\section{Statistical reviews}

SPSS (Statistical Package for Social Sciences) for Windows 16.0 software package was used for statistical analysis. While the data was analyzed comparison between groups of parameters normal distribution for the comparison of quantitative data Student $t$ test was used as well as descriptive statistical methods (mean, standard deviation, median), and in comparisons between groups of parameters that are normally distributed, Mann-Whitney $\mathrm{U}$, Wilcoxon's rank sum test was used. Results were evaluated at $95 \%$ confidence interval and the significance was evaluated at $p<0.05$ level.

\section{RESULTS}

In this study, the age of the patients ranged from 18 to 65 and an average was $49.11 \pm 13.89$ years and 141 men and 160 women participated in the study. Degree of unilateral hearing loss was 31 slight, 64 moderate, 49 moderately severe, 35 severe and 8 profound. In the ear in which hearing aid was used, there was 134 sensorineural hearingloss, 53 mixture hearing loss. Degree of hearing loss of the people using hearing aid bilaterally was 24 slight, 79 moderate, 54 moderately severe, 48 severe and 23 profound. In 162 ears of people using hearing aid bilaterally there was sensor neural hearing loss and in 53 ears there was mixture hearing loss. Daily device usage time of the patients ranged between 3-20 hours and was avarage 13 hours. On the assessment subscale scores on the use of the right or left ear hearing aids, there is not a statistically significant difference between the scores 
obtained from EC, BN, RV and AV subscales ( $p>0.05)$. The minimum, maximum and average values obtained from using right and left ear hearing aid are shown in Table 1.

When we analyze in terms of using hearing aid in one ear or in both ears; minimum, maximum and average values obtained are shown in Table 2. Individuals using hearing aid in both ears got higher scores than individuals using hearing aid in one ear for 3 sub- scale groups (EC, BN, RV) and there has been a statistically important significance between the average scores of individuals using hearing aid in both ears and in single ear $(p<0,05)$. But in Adoption of Voice subscale (AV) there hasn't been a statistically important significance between the scores of individuals using hearing aid in both ears and in single ear $(p<0,05)$, (Table 2). In the scores of individuals who use the hearing aid in their right ear a decline was observed as their loss of hearing increases from slight to profound. The general satisfaction score average of individuals having slight hearing loss in right ear is 5.75; satisfaction score average of individuals having moderate hearing loss is 5.00; satisfaction score average of individuals having moderate severe hearing loss is 4.83; satisfaction score average of individuals having severe hearing loss is 4.50 and average satisfaction score of individuals having profound hearing loss is 4.23. As the degree of hearing loss of individuals using hearing aid in their right ear is increasing, the scores they get are decreasing and there has been a statistically

Table 1: The comparison of subscale values that are used with hearing aids in the right and left ear.

\begin{tabular}{llcccc}
\hline & & EC & BN & RV & AV \\
\hline \multirow{4}{*}{ Right } & $\mathbf{n}$ & 102 & 100 & 102 & 101 \\
& Minimum & 2.5 & 1.83 & 2 & 3.17 \\
& Maximum & 7 & 6.67 & 7 & 7 \\
& Average & 4.41 & 4.33 & 4.41 & 6.66 \\
& $\mathbf{n}$ & 85 & 85 & 85 & 85 \\
Left & Minimum & 2.5 & 2.17 & 3 & 3.17 \\
& Maximum & 7 & 7 & 6.83 & 7 \\
& Average & 4.66 & 4.66 & 4.5 & 6.66 \\
p value & & 0.537 & 0.243 & 0.664 & 0.115 \\
\hline
\end{tabular}

Table 2: Comparison the sub-scale values due to using of single ear or both ears hearing aid.

\begin{tabular}{llcccc}
\hline & & EC & BN & RV & AV \\
\hline \multirow{4}{*}{ Single ear } & $\mathbf{n}$ & 187 & 185 & 187 & 186 \\
& Minimum & 2.5 & 1.83 & 2 & 3.17 \\
& Maximum & 7 & 7 & 7 & 7 \\
& Median & 4.5 & 4.5 & 4.5 & 6.66 \\
Double ear & $\mathbf{n}$ & 114 & 114 & 114 & 114 \\
& Minimum & 3 & 3 & 2.5 & 1 \\
& Maximum & 7 & 7 & 7 & 7 \\
p value & Median & 5.83 & 5.66 & 5.66 & 6.16 \\
\hline
\end{tabular}

significant difference between these scores $(p<0.05)$. In the result of comparison of sub-scale value with the right ear hearing loss degrees in EC, BN and RV there has been a statistically significant results while $(p<0.05)$ in AV subscale there has not been a statistically significant result $(p>0.05)$. A decrease has been observed in the scores of individuals using hearing aid in their left ears as their hearing loss increasing from slight to profound. The general satisfaction score average of individuals having slight hearing loss in left ear is 5.72; satisfaction score average of individuals having moderate hearing loss is 5.04; satisfaction score average of individuals having moderate - severe hearing loss is 4.83; satisfaction score average of individuals having severe hearing loss is 4.37 and average satisfaction score of individuals having profound hearing loss is 4.20 . As the degree of hearing loss of individuals using hearing aid in their left ear is increasing, the scores they get are decreasing and there has been a statistically significant difference between these scores $(p<0.05)$. In the result of comparison of subscale value with the right ear hearing loss degrees in $\mathrm{EC}$, $\mathrm{BN}$ and $\mathrm{RV}$ there has been a statistically significant results while $(p<0.05)$ in AV subscale there has not been a statistically significant result ( $p>0.05)$. In terms of having hearing aids in right ear or in left ear there hasn't been a statistically significant difference according to the type of hearing loss and on the basis of sub-scales ( $p>0.05)$. When it is evaluated in terms of age, users of hearing aids between the age of 18 and 60 have 5 or above average scores; it is seen that general average decreases to 4.83 for the users between the age of 60 and 65 . For 18-60 years old and 60-65 years old hearing aid users there has been statistically significant difference in the BN and RV subscales $(p<0.05)$, while a significant difference could not found in EC and AV subscales ( $p>0.05)$.

\section{DISCUSSION}

Priority objective in the management of patients with hearing loss is to make the use of hearing aids acceptable to eliminate the barriers of hearing loss. For this the time passes until the patient begins using hearing aid should be used ideally. It is necessary to make the audio logical research and hearing aid implementation fittingly. Evaluating the benefits of hearing aids with REM objectively and checking the hearing aids and accessories (mold, battery, etc.) at regular intervals will improve patient satisfaction. Early detection of hearing loss, early acceptance and to start using hearing aids in time increases the success and satisfaction of the hearing aid device in a positive way. There are many factors that affect success and satisfaction. Patient's expectation from the aid, psychological and social factors, the cost of hearing aids, general health issues, the physical properties of hearing aids and cosmetic issues created by them, the acoustic characteristics of hearing aids (sound quality) are important factors ${ }^{10,11}$. Patient satisfaction can also be measured with the surveys used 
on the patients. In this study, APHAB-TR questionnaire was conducted to evaluate hearing aid satisfaction of users. There is an increase in speech discrimination scores with the usage of hearing aids. But these tests conducted in a quiet environment and ideal conditions may not always reflect reality. Because of this, in quiet and noisy environment it is possible to evaluate patient satisfaction with questionnaires ${ }^{12-14}$. When the subscales of the APHAB-TR questionnaire are taken into account; $E C, R V$ and $B N$ shows the benefits at the international level in a realistic manner. However, compliance with the AV subscale scores may not reflect the overall benefits. It is also possible to carry out a satisfaction evaluation with such as; Satisfaction with Amplification in Daily LifeSADL, International Outcome Inventory for Hearing AidsIOIHA, Glasgow Hearing Aid Benefit Profile- GHABP etc. except from APHAB questionnaire. Our study is the first satisfaction survey done with $A P H A B-T R$ questionnaire in our country. Before the questionnaire was administered to the patients, the Real-Ear Measurements (REM) was performed with hearing aids. Therefore, all the negativity that may arise from accessories such as hearing aids and mold was removed. To eliminate the negativity connected to the aid or accessory provided to focus on the satisfaction completely. In the study they conducted evaluated the satisfaction of users of analogue and digital hearing aids by using "APHAB" questionnaire. Adaptation of devices using analogue equipment is $43 \%$ and in users of digital hearing aids it is $82 \%$. This study shows that developing hearing aid technology facilitates the adaptation of devices ${ }^{15}$. Suggested that normative data of GHABP survey on 1574 adults with profound hearing loss and have expressed that it can be used reliably in hearing aid satisfaction evaluation ${ }^{16}$ in their study found out that individuals with hearing aid who got consultancy service after pre and post fitting have higher user satisfaction compared to those who didn't get consultancy with GHABP questionnaire ${ }^{17}$ recommended the use of bilateral hearing aids in their study in which they evaluated the satisfaction of the individuals using featured digital hearing aids by using SADL questionnaire. Those devices which are technologically advanced showed satisfaction from the aid by using SADL questionnaire in demanding environments and background noise ${ }^{18}$. In our study we indicated with $A P H A B$ questionnaire that the satisfaction obtained from the use of bilateral hearing aids more than one-sided hearing aid. Consequently, we like suggest using bilateral hearing aids. satisfaction evaluation on 100 patients and demonstrated with IOI-HA-TR questionnaire that $80 \%$ of patients use their hearing aid more than four hours per day, $64 \%$ of which benefit significant benefits from the device use, $67 \%$ of which have no problems or very few problems according to the pre-device period. Also $77 \%$ of the cases stated that after using the hearing aid their existing hearing loss didn't affect their work or affected slightly ${ }^{19}$ indicated in their study conducted with IOI-HA questionnaire that $90 \%$ of patients got moderate or more benefits. $97 \%$ of the patients' quality of life, showed markable improvement. These results indicated that patients had substantial satisfaction and significant improvement in quality of their life from the usage of hearing aids ${ }^{20}$. It evaluated the satisfaction with "APHAB" questionnaire at $3^{\text {rd }}, 6^{\text {th }}$ and $12^{\text {th }}$ months after only hearing instruments and Electro-Acoustic Stimulation (EAS). By using only hearing aid showed $74 \%$ reduction in hearing loss. 3 months later in the satisfaction evaluation after EAS applications they reported $45 \%$ improvement in hearing rate $^{21}$ reported high satisfaction obtained from bone implants done to15 adult patients with bilateral ear atresia. They received high satisfaction scores from $\mathrm{EC}, \mathrm{RV}$ and $\mathrm{BN}$ subscales of $\mathrm{APHAB}$ and indicated that they performed communication more comfortably when compared to scores of communication without aids ${ }^{22}$ made satisfaction assessment on patients with cochlear implants. In this study how to eliminate negative effects of telephone usage, to chat with others, communication skills and hearing loss of the patients who use cochlear implants 16 hours in a day with SADL questionnaire was shown ${ }^{23}$ stated in the study which was done with $A P H A B$ that questionnaires form the $3^{\text {rd }}$ part of the evaluation the hearing aid satisfaction evaluation after the pure tone and speech testing. But they could not find a correlation between AV scale and ANL (Acceptable Noise Levelsacceptable noise level) ${ }^{24}$ found a slight correlation between pure tone and speech test measurements and hearing loss indicated by individual subjectively and disability in their study ${ }^{25,26}$. Especially patients with slight and mild hearing loss showed major differences in terms of hearing loss ${ }^{27,28}$. Showed in their study in which they evaluated satisfaction in terms of type of the hearing loss and degree done with SADL questionnaire that there has been a decrease of the satisfaction of patients having severe and profound hearing loss ${ }^{29}$. In our study it is observed that while degree of hearing loss is increasing from slight to profound, there occurs a decrease in satisfaction. Results similar to have been obtained In our study average usage duration of the aid users is 13 hours a day and they have experience of at least 6 weeks. It has been considered that patients have satisfaction owing to long usage duration. In the studies it has been shown that benefit of hearing aid and decrease of hearing loss is high due to regular usage of hearing aid in a certain period of time ${ }^{27-31}$. Auditory centre in the brain is located in the left temporal lobe. Because of the fact that installing the hearing aid to right ear due to cerebral lateralization and the actual crossing of the left temporal lobe will stimulate more comfortably; therefore, it is thought that it is suitable to use the aid in right ear in order to understand speech more comfortably ${ }^{32}$. In the study we have done it has been shown that there is no advantage to use the aid in right ear or in the left ear in terms of satisfaction. Because of this reason if the aid is used in one ear, either right ear or left ear can be preferred. As a result; hearing aids should be recommended to patients bilaterally. If the patient will 
use single device there is no difference in the selection of right or left ear.

\section{CONCLUSION}

Type of hearing loss does not affect the satisfaction obtained from the hearing aid. Patients with hearing loss should start using hearing aids from the moment of hearing loss occurs. Satisfaction is reduced at 60 years of age and over. Therefore, to get used to hearing aid usage before 60 years of age is extremely important for the future benefit and satisfaction.

\section{CONFLICT OF INTEREST}

Author declares no competing interests.

\section{REFERENCES}

1. Plack CJ. Hearing impairment. In: Oxford Handbook of Auditory Science: Hearing. Oxford: Oxford University Press. 2010.

2. Brown AD, Beemer BT, Greene NT, Argo T, Meegan GD, Tollin DJ. Effects of Active and Passive Hearing Protection Devices on Sound Source Localization, Speech Recognition, and Tone Detection. PLoS One. 2015;27:1365-8.

3. Zhao F, Bardsley B. Real-ear acoustical characteristics of impulse sound generated by golf drivers and the estimated risk to hearing: a cross-sectional study. BMJ Open. 2014;4:351-7.

4. Kaplan H, Bally S, Brandt F, Busacco D. Pray Journal of Communication Scale for Older Adults (CSOA). J Am Acad Audiol. 1997;8:203-17.

5. Judy L, Huch MS, Holly HD. Inventories of Self-Assessment Measurements of Hearing Aid Outcome, In: Sandlin RE. Hearing Aid Amplification, Technical and Clinical Considerations, $2^{\text {nd }}$ Edition Singular Publishing Group, San Diego, California. 2000:489-519.

6. Cox RM, Alexander GC. The abbreviated profile of hearing aid benefit. Ear Hear. 1995;16:176-86.

7. Marchesin VC, lório MC. Study of the long-term effects of frequency compression by behavioral verbal tests in adults. Codas. 2015;27:37-43.

8. Reinfeldt S, Håkansson B, Taghavi H, Fredén Jansson KJ, Eeg-Olofsson $\mathrm{M}$. The bone conduction implant: Clinical results of the first six patients. Int J Audiol. 2015;54:408-16.

9. Kam AC, Tong MC, Van Hasselt A. Cross-cultural adaptation and validation of the Chinese abbreviated profile of hearing aid benefit. Int J Audiol. 2011;50:334-9.

10. Hosford-Dunn H, Halpern J. Clinical application of the satisfaction with amplification in daily life scale in private practice I: statistical, content, and factorial validity. J Am Acad Audiol. 2000;11:523-39.

11. Baumfield A, Dillon $H$. Factors affecting the use and perceived benefit of ITE and BTE hearing aids. $\mathrm{Br} \mathrm{J}$ Audiol. 2001;35:247-58.

12. Bille $M$, Parving $A$. Expectations about hearing aids: demographic and audiological predictors. Int $\mathrm{J}$ Audiol. 2003;42:481-8.

13. Stephens D. The International Outcome Inventory for Hearing
Aids (IOI-HA) and its relationship to the Client-oriented Scale of Improvement (COSI). Int J Audiol. 2002;41:42-7.

14. Johnson CE, Danhauer J. The 'Hearing Aid Effect' revisited: Can we achieve hearing solutions for cosmetically sensitive patients. In: Kochkin KE, High performance Hearing Solutıons. Supplement to The Hearing Review. 1997;1:3744.

15. Johnson JA, Cox RM, Alexander GC. Development of APHAB norms for WDRC hearing aids and comparisons with original norms. Ear Hear. 2010;31:47-55.

16. Whitmer WM, Howell P, Akeroyd MA. Proposed norms for the Glasgow Hearing-Aid Benefit Profile (GHABP) questionnaire. Int J Audiol. 2014;53:345-51.

17. Kemker BE, Holmes AE. Analysis of prefitting versus postfitting hearing aid orientation using the Glasgow Hearing Aid Benefit Profile (GHABP). J Am Acad Audiol. 2004;15:311-23.

18. Kaplan-Neeman R, Muchnik C, Hildesheimer M, Henkin Y. Hearing aid satisfaction and use in the advanced digital era. Laryngoscope. 2012;122:2029-36.

19. Hamurcu M, Sener BM, Atas A, Atalay RB, Bora F, Yigit O. Evaluation of Satisfaction in Patients with Hearing Aids. KBBForum. 2012;11:26-31.

20. Cook JA, Hawkins DB. Outcome measurement for patients receiving hearing aid services. Laryngoscope. 2007;117:61013.

21. Gstoettner WK, Van de Heyning P, Oconnor AF. Assessment of the subjective benefit of electric acoustic stimulation with the abbreviated profile of hearing aid benefit. ORL $J$ Otorhinolaryngol Relat Spec. 2011;73:321-9.

22. Yue F, Yibei W, Zhen W, Pu W, Xiaowei C. Self-rated efficacy in bilateral aural atresia patients using bone-anchored hearing aid. 2015;50:203-9.

23. Faber HT, Nelissen RC, Kramer SE, Cremers CW, Snik AF, $\mathrm{Hol} \mathrm{MK}$, et al. Bone-anchored hearing implants in singlesided deafness patients: Long-term use and satisfaction by gender. Laryngoscope. 2015;125:2790-5.

24. Löhler J, Akcicek B, Kappe T, Schlattmann P, Wollenberg $B$, Schönweiler R. Development and use of an APHAB database. HNO. 2014;62:735-45.

25. Demorest ME, Walden BE. Psychometric principles in the selection, interpretation, and evaluation of communication self-assessment inventories. J Speech Hear Disord. 1984;49:226-40.

26. Taylor KS. Self-perceived and audiometric evaluations of hearing aid benefit in the elderly. Ear Hear. 1993;14:390-4.

27. Newman CW, Weinstein BE. The Hearing Handicap Inventory for the Elderly as a measure of hearing aid benefit. Ear Hear. 1988;9:81-5.

28. Weinstein BE, Ventry IM. Audiometric correlates of the Hearing Handicap Inventory for the elderly. J Speech Hear Disord. 1983;48:379-84.

29. Mondelli MF, Rocha AV, Honório HM. Degree of satisfaction among hearing aid users. Int Arch Otorhinolaryngol. 2013;17:51-6.

30. Mulrow CD, Aguilar C, Endicott JE, Tuley MR, Velez R, Charlip 
WS, et al. Quality-of-life changes and hearing impairment. A randomized trial. Ann Intern Med. 1990;113:188-94.

31. Cox RM, Alexander GC. The International Outcome Inventory for Hearing Aids (IOI-HA): psychometric properties of the
English version. Int J Audiol. 2002;41:30-5.

32. Bidelman GM, Howell M. Functional changes in intrahemispheric cortical processing underlying degraded speech perception. Neuro image. 2016;124:581-90. 